\title{
Single-molecule fluorescence studies on the conformational change of the $A B C$ transporter MsbA
}

\author{
Yanqing Liu ${ }^{1,2}$, Yue $\mathrm{Liu}^{1}$, Lingli $\mathrm{He}^{1}$, Yongfang Zhao ${ }^{1}$, Xuejun C. Zhang ${ }^{1,2 \bowtie}$ \\ 1 National Laboratory of Biomacromolecules, National CAS Center for Excellence in Biomacromolecules, Institute of \\ Biophysics, Chinese Academy of Sciences, Beijing 100101, China \\ 2 College of Life Science, University of Chinese Academy of Sciences, Beijing 100049, China
}

Received: 2 April 2018 / Accepted: 11 April 2018 / Published online: 12 June 2018

\begin{abstract}
ATP-binding cassette $(\mathrm{ABC})$ transporters are found in all forms of life from microbes to humans, and transport a wide variety of substrates across the cell membrane using the energy released from ATP hydrolysis and an alternating-access mechanism. MsbA is a homodimeric ABC exporter from Gramnegative bacteria, and transports amphipathic substrates including precursors of lipopolysaccharides from the inner leaflet to the outer leaflet of the cytoplasmic membrane. Despite extensive structural and functional studies, controversies remain regarding the dynamic properties of the conformational changes of MsbA during its transport cycle in the lipid environment. Here, we used single-molecule fluorescence resonance energy transfer (smFRET) to explore the dynamic behaviors of MsbA in detergent micelles, nanodiscs, and proteoliposomes. MsbA reconstituted into liposomes showed higher transition frequency between different states on the cytoplasmic side, whereas detergent-solubilized MsbA showed higher transition frequency on the periplasmic side. Three major states were identified from this smFRET study in the functional cycle of MsbA, including an intermediate conformation between the fully opened and fully closed cytoplasmic conformations, associated with both ATP binding and hydrolysis.
\end{abstract}

Keywords ABC transporter, Conformational change, Single-molecule fluorescence resonance energy transfer (smFRET)

\section{INTRODUCTION}

Adenosine triphosphate (ATP)-binding cassette (ABC) transporters are a large superfamily of membrane proteins that actively translocate a wide variety of substrates across the cellular membrane, driven by energy released from ATP hydrolysis (Dassa 2011; Davidson and Chen 2004; Higgins 2007; Locher 2016; Zhang et al. 2016). All ABC transporters share a common molecular

Electronic supplementary material The online version of this article (https://doi.org/10.1007/s41048-018-0057-z) contains supplementary material, which is available to authorized users.

$\triangle$ Correspondence: zhangc@ibp.ac.cn (X. C. Zhang) architecture containing two transmembrane domains (TMDs) and two cytoplasmic nucleotide-binding domains (NBDs). The TMDs, which show high sequence diversity, form the translocation pathway and assume the role of substrate selectivity, whereas the NBDs are highly conserved and contain the ABC-characteristic motifs that bind ATP and catalyze its hydrolysis (Ward et al. 2007). $\mathrm{ABC}$ transporters are classified into importers and exporters depending on the direction of substrate translocation (Zhang et al. 2016).

MsbA from Gram-negative bacteria is an extensively studied ABC exporter (Doerrler and Raetz 2002). This $128-\mathrm{kDa}$ homodimeric transporter is located in the cytoplasmic (inner) membrane and plays a critical role in flipping Lipid A, a precursor of lipopolysaccharides, a 
major component of the bacterial outer membrane, from the intracellular side to periplasmic side of the inner membrane (Doerrler et al. 2001, 2004; Ruiz et al. 2009; Zhou et al. 1998). In addition, numerous hydrophobic/amphipathic small molecules can be transported by MsbA, and thus MsbA functions as a multidrug-resistance transporter. In its functional cycle, MsbA alternates between intracellular (inward)-facing and periplasmic (outward)-facing conformations, coupling ATP hydrolysis with large conformational rearrangements of the NBD dimer as well as the TMDs (Dong et al. 2005; Higgins and Linton 2004; Ward et al. 2007; Zou et al. 2009). Crystal structures and cryoelectron microscopy (cryo-EM) structures of MsbA have been reported in multiple conformational states, including an intracellular-opening apo state, an intracellular semiclosed apo state, an intracellular-closed state bound with a non-hydrolyzable ATP analog, and an intracellular-closed state in complex with both ADP (from ATP hydrolysis) and inorganic vanadate $\left(\mathrm{V}_{\mathrm{i}}\right)(\mathrm{Mi}$ et al. 2017; Ward et al. 2007).

In the last decade or so, a variety of techniques have been used to study the mechanism of the transport cycle of MsbA, such as EM (Moeller et al. 2015), chemical crosslinking (Doshi et al. 2010), double electronelectron resonance (Borbat et al. 2007; Zou et al. 2009; Zou and McHaourab 2010), fluorescence homotransfer (Borbat et al. 2007), and luminescence resonance energy transfer (Cooper and Altenberg 2013). However, different results were obtained, and the mechanism remains to be firmly consolidated. Moreover, membrane reconstitution (Rigaud et al. 1995), which in principle provides the target proteins with a microenvironment closer to the native membrane, has accelerated mechanistic studies of membrane proteins, including MsbA. However, there is still a lack of direct comparison of the effects of different reconstitution methods on the dynamics of transporters in general, and of MsbA in particular.

In the current study, we used the single-molecule fluorescence resonance energy transfer (smFRET) technique to study the conformational changes of MsbA in the cycle of ATP hydrolysis under three different conditions, namely in detergent micelles, nanodiscs, and liposomes. smFRET is a powerful tool for probing conformational changes in proteins with fairly high spatiotemporal resolution (Lerner et al. 2018), and it has been applied to the study of conformational dynamics of membrane proteins (Zhao et al. 2010a, b, 2011). Our smFRET data, monitored from the cytoplasmic side, showed that the population distribution of MsbA conformations is more restricted in nanodiscs than in either liposomes or micelles of the detergent n-dodecyl-Dmaltopyranoside (DDM). In addition, the transition frequency between distinct conformations of MsbA NBDs in liposomes is higher than that in either nanodiscs or DDM, suggesting that the NBD dimer is more mobile in the more native-like membrane environment than in the other two model systems. In contrast, the DDM micelles favor conformational changes of the TMD dimer of MsbA on the periplasmic side. Moreover, the ensemble of MsbA in the presence of ATP and $V_{i}$, which is believed to mimic the transition state of ATP hydrolysis, in fact represents a terminal-state that cannot be reached by simultaneous addition of ADP and $V_{i}$. These results may shed new light on mechanisms of the ABC transporter MsbA.

\section{RESULTS}

\section{Experimental design for smFRET analysis}

smFRET experiments were performed on variants of Escherichia coli MsbA containing a single cysteine (Cys) residue in each subunit linked to fluorophores by maleimide chemistry. Two wild-type (WT) Cys residues were first substituted with Ala, and the resulting Cysless MsbA (denoted WT*) was used as the template to construct single-Cys mutants. To probe conformational changes of MsbA, we individually mutated several sites to Cys based on available structural information. From these constructs, variants D277C (in the periplasmic region of the TMD) and T561C (in the cytoplasmic NBD) (Fig. 1A) were selected on the basis of their high labeling efficiency (>90\%), low nonspecific labeling (Supplemental Fig. S1A), and low anisotropy (Supplemental Table S1).

For the detergent-micelle assays, smFRET experiments were performed with recombinant MsbA variants which were $N$-terminally His-tagged and immobilized onto biotin-NTA-coated microfluidic channels in $0.05 \%$ DDM (Fig. 1B). To better mimic the membrane environment, we further performed smFRET experiments with MsbA proteins reconstituted into either liposomes or nanodiscs, both of which were made from $E$. coli total lipids, and the reconstituted proteins were surface immobilized through biotin-labeled lipids to streptavidin-decorated channels (Fig. 1B). The average diameter of the nanodiscs was estimated by EM imaging to be $\sim 12 \mathrm{~nm}$, and the liposome diameter was estimated to be $\sim 100 \mathrm{~nm}$. All the three preparations showed specific surface attachment (Supplemental Fig. S1B). ATPase activities of recombinant MsbA variants were measured in the three conditions. Results from the activity assays revealed that all the MsbA variants in nanodiscs showed significantly higher ATPase activity than those in either liposomes or DDM (Fig. 1C). 
A

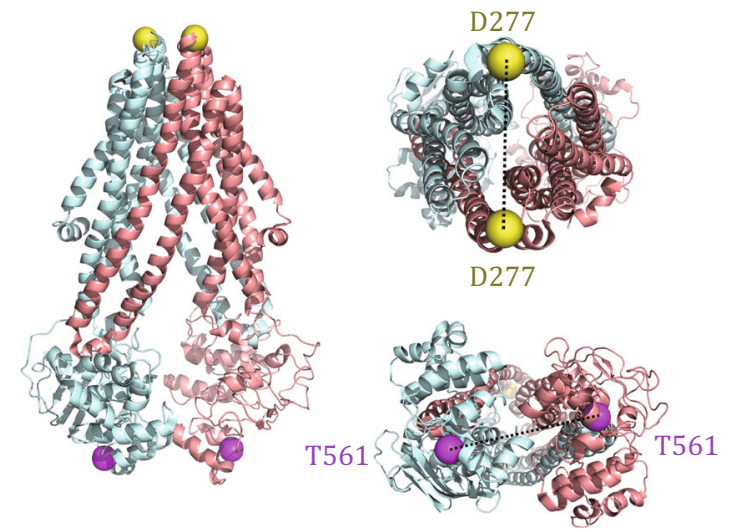

C

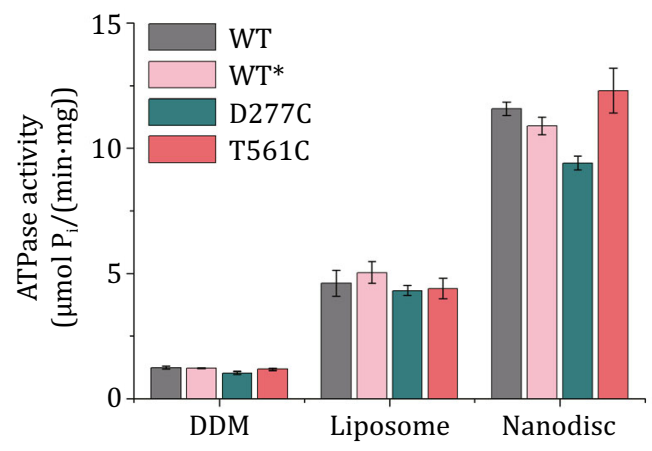

B

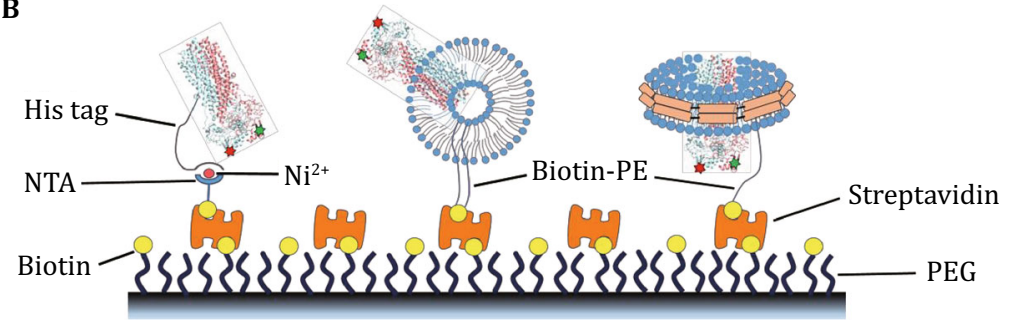

Fig. 1 Design of the smFRET experiments and ATPase activities of MsbA variants. A Cartoon representation of the cryo-EM structure of apo-MsbA (PDB ID: 5TV4). Positions of residues D277 and T561 are shown as spheres. B Schematic diagram of strategies to immobilize MsbA. C ATPase activities of wild-type (WT), Cys-less (WT*), D277C, and T561C variants of MsbA in DDM micelles, liposomes, and nanodiscs, respectively. In liposomes, the activities have been corrected for the fraction of protein (53\%) with an outward-facing orientation of the nucleotide-binding domains (NBDs). Error bars indicate standard deviations of results from three independent experiments

For instance, the ATP-hydrolysis rate of WT MsbA in nanodiscs $\left(24.9 \pm 2.6 \mathrm{~s}^{-1}\right.$ per MsbA molecule) was higher than that in DDM $\left(2.6 \pm 0.2 \mathrm{~s}^{-1}\right)$ or in liposomes $\left(10.0 \pm 0.8 \mathrm{~s}^{-1}\right)$, probably due to the nanodisc restricting MsbA to a conformation(s) favoring ATP hydrolysis. As expected, vanadate, which is an analog of inorganic phosphate $\left(\mathrm{P}_{\mathrm{i}}\right)$ and is believed to mimic the $\gamma$ phosphate of ATP in the transition state of ATP hydrolysis (Davidson 2002; Smith and Rayment 1996; Urbatsch et al. 2003), inhibited the ATPase activities of all of the MsbA variants used in this study (Supplemental Fig. S2B). The variation in activity in different solubilization conditions is consistent with previous studies. For example, in the previously reported nanodisc-embedded structure of MsbA, the two NBDs of MsbA assume a perfect dimer orientating the Walker A and signature motifs to form a NBD-ATP-NBD sandwich for ATP hydrolysis, and the ATPase activity of the corresponding recombinant protein was estimated to be $14 \mathrm{~s}^{-1}$ (Mi et al. 2017). In contrast, detergent-solubilized MsbA molecules assumed a cytoplasmic-opened conformation in the crystal structures (Ward et al. 2007) and had an ATPase activity of $2 \mathrm{~s}^{-1}$ (Eckford and Sharom 2008).

\section{Conformational changes of NBDs}

\section{Population distribution in different states}

To study conformational changes of the MsbA NBDs during the ATP-hydrolysis cycle, the T561C variant was chosen to probe the smFRET signals with different ligand combinations. The mutation site, T561C, is located on the membrane-distal surface of the NBD. In the nanodiscs, the nucleotide-free (apo) state mainly produced a unimodal distribution, peaking at $\sim 0.6$ FRET efficiency ( $E_{\text {FRET }}$, the value of which ranges between 0.0 and 1.0) (Fig. 2A), consistent with the cryo-EM structure of apo-MsbA in which the $\mathrm{C}_{\alpha}$ $\mathrm{C}_{\alpha}$ distance between the two T561 residues is $\sim 35 \AA$ (Mi et al. 2017). In both ADP (5 mmol/L) and ADP (5 mmol/L)- $V_{\mathrm{i}}(1 \mathrm{mmol} / \mathrm{L})$ ensembles, the MsbA population also showed a similar $E_{\text {FRET }}$ distribution (Fig. 2A), indicating that binding of ADP either alone or with additional $\mathrm{V}_{\mathrm{i}}$ did not promote conformational change in MsbA. In contrast, in the presence of either ATP (5 mmol/L) or the non-hydrolyzable ATP analog $\beta, \gamma$-imido-adenosine $5^{\prime}$ triphosphate (AMPPNP, $5 \mathrm{mmol} / \mathrm{L}$ ), MsbA displayed an $E_{\text {FRET }}$ peak at $\sim 0.65$ (Fig. $2 \mathrm{~A}$ ), revealing a slightly closer 
A

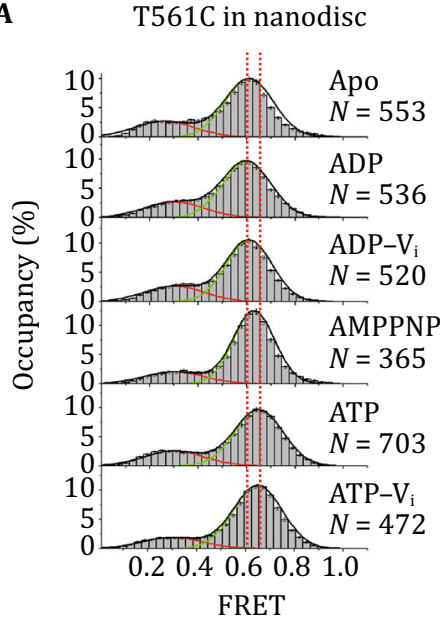

B

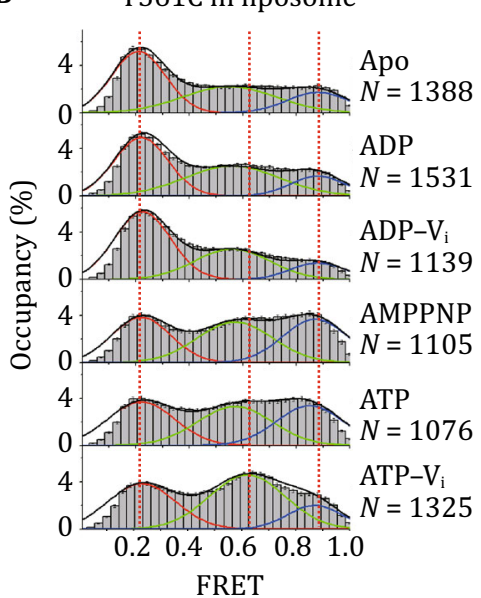

C

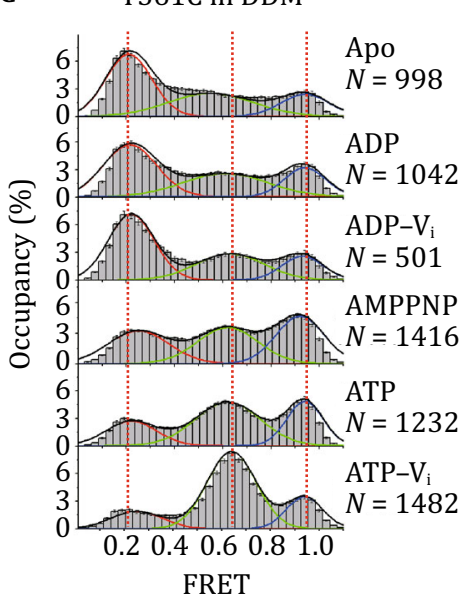

D
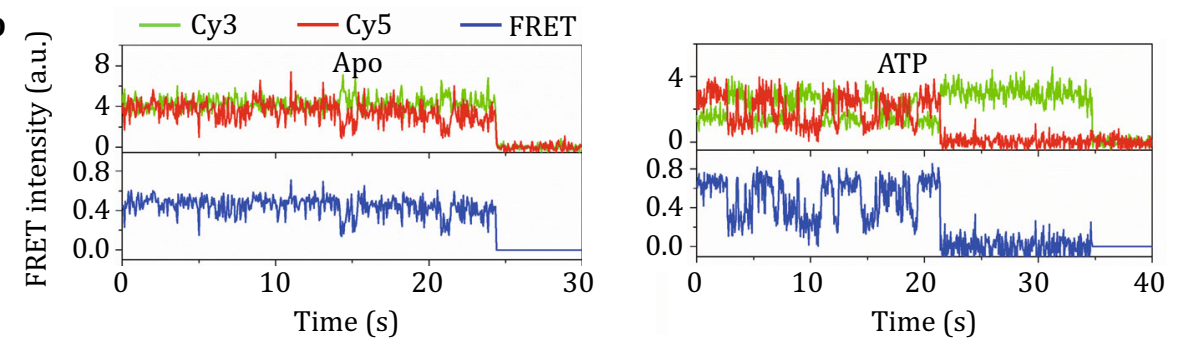

E

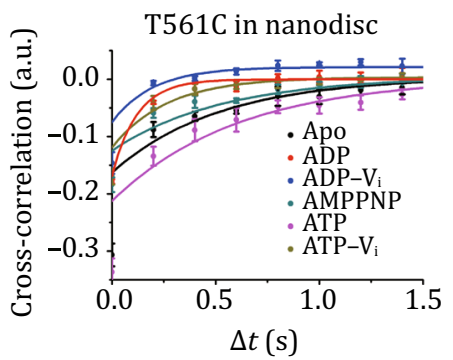

F
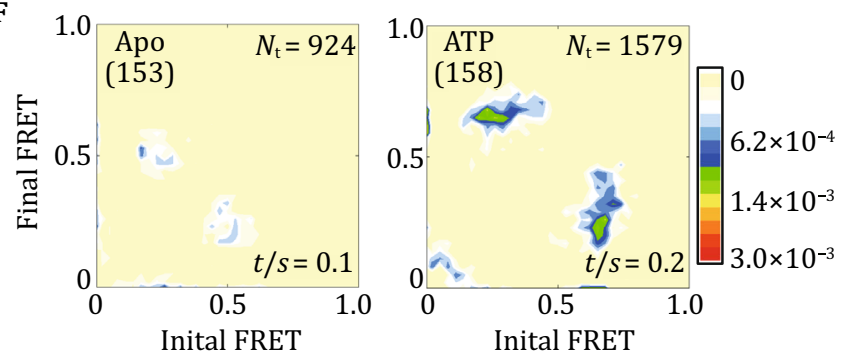

Fig. 2 Conformational dynamics of MsbA NBDs. A-C Fluorescence resonance energy transfer (FRET) histograms for MsbA-T561C reconstituted into nanodiscs (A), liposomes (B), and in n-dodecyl-D-maltopyranoside (DDM) (C), in different ligand-binding ensembles. $N$, the number of MsbA molecules included in the analysis. Error bars indicate standard deviations of 1000 bootstrap samples of the FRET traces. D Representative single-molecule fluorescence trajectories from the apo and ATP ensembles of MsbA-T561C in nanodiscs. E Crosscorrelation analysis of fluorescence trajectories between donors and acceptors in the nanodiscs. The ATP ensemble showed a stronger negative correlation than other ensembles, indicating that MsbA molecules are more dynamic in this ensemble. Trajectories from all experimental donor-acceptor pairs were used in the calculation. Error bars indicate standard deviations of results from five independent experiments. F Transition-density plots of the apo and ATP ensembles of MsbA-T561C in nanodiscs. MsbA molecules undergoing recognizable transitions were manually selected to make the transition-density plots (the number of selected molecules is shown in parentheses), and three-state transitions were assumed. $N_{t}$ is the number of total transitions included in the analysis; $t / s$ is the average transition frequency per MsbA molecule. The color scale of the transition rate is from light-yellow (background, zero) to red (most populated)

arrangement between the two NBDs than in the apo state. To trap MsbA molecules in their ATP-hydrolysis transition state, we added vanadate together with ATP to the smFRET assay. Like ATP and AMPPNP, addition of ATP $(5 \mathrm{mmol} / \mathrm{L})-\mathrm{V}_{\mathrm{i}}(1 \mathrm{mmol} / \mathrm{L})$ promoted a population shift toward higher $E_{\mathrm{FRET}}$ for MsbA-T561C (Fig. 2A).
Sharply distinct from nanodiscs, the proteoliposome of apo-MsbA displayed an intracellular-opening conformation with a unimodal distribution at $E_{\mathrm{FRET}} \sim 0.2$ (Fig. 2B). In liposomes, both the ADP and ADP-V ensembles showed an $E_{\mathrm{FRET}}$ distribution similar to the apo state (Fig. 2B), again demonstrating that binding of either ADP or ADP- $V_{i}$ does not stimulate NBD 
dimerization. Similar results were observed in DDM micelles (Fig. 2C), in agreement with previous spectroscopic studies and the crystal structure of detergentsolubilized apo-MsbA (Moeller et al. 2015; Ward et al. 2007; Zou et al. 2009). More importantly, under both liposome and DDM conditions, the $E_{\mathrm{FRET}}$ distribution of the AMPPNP ensemble revealed a broad profile with one peak at about 0.9 , corresponding to an intracellularclosed conformation, and a similar FRET distribution was observed for the ATP ensemble (Fig. 2B, C). Interestingly, the $E_{\mathrm{FRET}}$ distribution showed a peak at $\sim 0.6$ for the ATP- $V_{i}$ ensemble, especially in DDM (Fig. 2B, C). These results demonstrated that, in liposomes and detergent micelles, binding of either AMPPNP or ATP thermodynamically favors NBD dimerization, resulting in two extra clusters of states of high (0.9) and intermediate (0.6) $E_{\mathrm{FRET}}$ values. In addition, in the presence of $V_{i}$, binding of ATP (which is subsequently converted to ADP) seems to particularly stabilize the intermediate$E_{\text {FRET }}$ state(s) that likely has a less compact NBD-dimer structure than the high- $E_{\mathrm{FRET}}$ state(s). An identical profile of $E_{\text {FRET }}$-population distributions in both liposomes and DDM, however, does not necessarily indicate the same underlying kinetics in state transitions, as shown below.

\section{Dynamics of conformational changes}

Using cross-correlation analysis (Vafabakhsh et al. 2015) on the donor- and acceptor-fluorescence data obtained from MsbA-T561C in nanodiscs, we found that the amplitude of negative correlation between donor- and acceptor-fluorescence trajectories in the ATP ensemble was larger than that in other ensembles (Fig. 2E). Since only transitions between drastically different FRET states contribute consistently to the negative correlation, the larger amplitude from the ATP ensemble likely reflects more frequent conformational transitions, presumably being stimulated by ATP hydrolysis. Furthermore, manual inspection of the FRET trajectories of different ligand-binding ensembles revealed that only a fraction (10\%-30\%) of MsbA molecules showed recognizable transitions before being photobleached. These data suggest the possibility that a given cluster of MsbA molecules of similar $E_{\mathrm{FRET}}$ occupy multiple states, and only some of these subpopulations are involved in frequent transitions with other clusters with distinct $E_{\text {FRET }}$ values. These MsbA molecules capable of transition were further analyzed using hidden Markov modeling (HMM) (McKinney et al.2006), resulting in estimates of the lower-limit of the dwell times $\left(\tau_{\text {dwell }}\right)$ of different clusters and the upperlimit of transition rates $(\kappa)$ between clusters. Indeed, peaks in the transition-density plot from the HMM analysis often showed minor yet recognizable shifts relative to the FRET-population distribution (e.g. Fig. 2F). Such shifts are likely to be related to insensitivity of the HMM method to transitions between states with only small differences in FRET values (McKinney et al. 2006). In the ATP ensemble, transitions between low- and intermediate- $E_{\mathrm{FRET}}$ states (with $E_{\mathrm{FRET}} \sim 0.3$ and $\sim 0.65$, respectively) occurred at an average rate of $\sim 0.2 \mathrm{~s}^{-1}$; the apo ensemble showed an average rate of $\sim 0.1 \mathrm{~s}^{-1}$ (Fig. 2D, F). Since a complete cycle of conformational changes requires at least two transitions, the average rate of such cycles in the ATP ensemble is estimated to be $0.1 \mathrm{~s}^{-1}$ or lower. Consistent with the pattern of transition rates, the dwell times in both the low- and intermediate$E_{\text {FRET }}$ states of apo-MsbA were $\sim 5$-times longer than those in the presence of ATP alone (Supplemental Fig. S2C).

Unlike in nanodiscs, in DDM micelles, MsbA NBDs showed a slightly stronger negative correlation in the apo ensemble than in the other ensembles (Supplemental Fig. S3B). Both inspection of the FRET trajectories of individual apo-MsbA molecules and the transitiondensity plot showed that, in DDM, conformational transitions of the apo ensemble mostly occurred between states with low (0.2) and intermediate (0.6) $E_{\mathrm{FRET}}$, with an average transition frequency of $\sim 0.3 \mathrm{~s}^{-1}$ (Supplemental Fig. S3A, C). These results suggested that, while it is mainly in the cytoplasmic-open state $\left(E_{\mathrm{FRET}} \sim 0.2\right)$, the NBD dimer of apo-MsbA may transiently visit the cytoplasmic semiclosed state $\left(E_{\mathrm{FRET}} \sim 0.6\right)$.

In liposomes, the MsbA NBDs showed fairly strong negative correlation between the donor- and acceptorfluorescence trajectories in all apo and ligand-binding ensembles (Fig. 3B), suggesting that MsbA molecules are more dynamic in liposomes than in nanodiscs. The trajectories from individual MsbA molecules and the transition-density plot showed frequent transitions between states of low-, intermediate-, and high-FRET, centering at $E_{\mathrm{FRET}}$ of about $0.2,0.6$, and 0.8 , respectively (Fig. 3A, C). For instance, in the apo ensemble, transitions mainly occurred between states with $E_{\text {FRET }} \sim 0.2$ and $\sim 0.6$, and only a small fraction of proteins underwent transition between 0.6 and 0.8 . The transitions (mainly between the states with $E_{\mathrm{FRET}} \sim 0.2$ and $\sim 0.6$ ) occurred at an average rate of $\sim 0.3 \mathrm{~s}^{-1}$, roughly three-times more frequent than in the nanodiscs. The ADP and ADP- $V_{i}$ ensembles showed dynamics similar to that of the apo ensemble. Therefore, apo-MsbA molecules mostly populate the intracellular-open conformations and "occasionally" assume the intracellular semiclosed conformation; neither ADP- nor ADP- $\mathrm{V}_{i}$-binding stimulates NBD dimeri zation. Furthermore, the transition-density plot of the AMPPNP ensemble showed frequent transitions between 

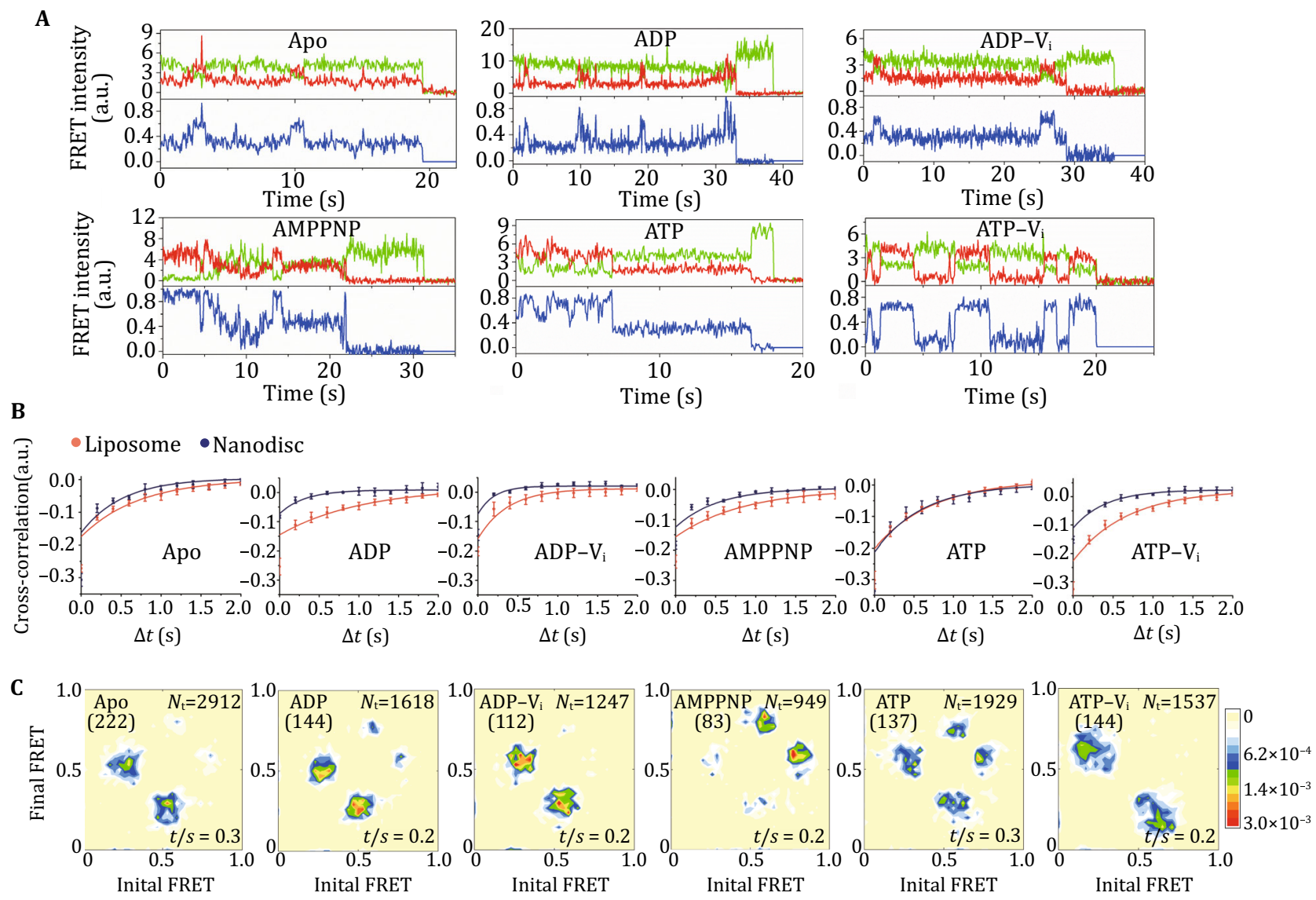

Fig. 3 Conformational dynamics of NBDs of MsbA in liposomes. A Representative single-molecule trajectories from the apo and different nucleotide ensembles of MsbA-T561C in liposomes. B Cross-correlation analysis of fluorescence trajectories between donors and acceptors in liposomes compared with nanodiscs. The results indicate that MsbA molecules in liposomes (orange curves) are more dynamic than those in nanodiscs (blue curves) in nearly all ligand-binding ensembles. Error bars indicate standard deviations of results from five independent experiments. C Transition-density plots of all ligand-binding ensembles of MsbA-T561C in liposomes. A three-state transition was assumed (See Fig. 2F for legend)

states with $E_{\text {FRET }} \sim 0.6$ and $\sim 0.8$ (Fig. 3C), and the dwell time was longer in the high-FRET state than in the other two states (Supplemental Fig. S2D). Thus, AMPPNPbinding induces dimerization of the NBDs and promotes the intracellular-closed conformation. The ATP ensemble showed a transition-density plot similar to that of the AMPPNP ensemble, with an extra transition between states with $E_{\text {FRET }} \sim 0.2$ and $\sim 0.6$ (Fig. $3 C$ ); the latter is probably associated with ATP hydrolysis, as suggested by $E_{\mathrm{FRET}}$ data in nanodiscs. Importantly, the transition-density plot of the ATP- $\mathrm{V}_{\mathrm{i}}$ ensemble showed frequent transitions, mainly between states with $E_{\text {FRET }} \sim 0.2$ and $\sim 0.6$ (Fig. 3C).

\section{Conformational changes of the TMDs}

We examined the conformational dynamics of the TMDs of the MsbA-D277C variant. Each TMD contains six transmembrane helices (TM1-TM6). Residue D277 is in a short periplasmic loop that connects TM5 and TM6. Patterns of conformational changes probed by a donoracceptor pair at the D277C sites were expected to be reciprocal to those of $\mathrm{T561C}$ if the movements of the cytoplasmic and periplasmic regions of MsbA were fully coupled.

In the nanodiscs, the apo ensemble produced a unimodal distribution peaking at $E_{\mathrm{FRET}} \sim 0.9$, and so did the ensembles with ADP and ADP-V $\mathrm{V}_{\mathrm{i}}$ (Fig. 4A). These data indicated that MsbA adopts a periplasmic-closed conformation in the apo ensemble, and neither binding of ADP nor ADP- $V_{i}$ affects this distribution. In the AMPPNP and ATP- $V_{i}$ ensembles, the $E_{\text {FRET }}$ population peak shifted toward lower $E_{\text {FRET }}$ of $\sim 0.8$ (Fig. $4 \mathrm{~A}$ ), suggesting that both ensembles had a less-compact conformation on the periplasmic side. Moreover, crosscorrelation analysis showed weak negative correlations 
A

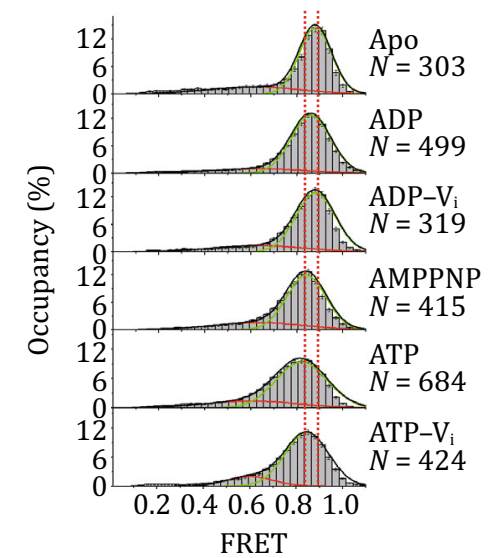

B

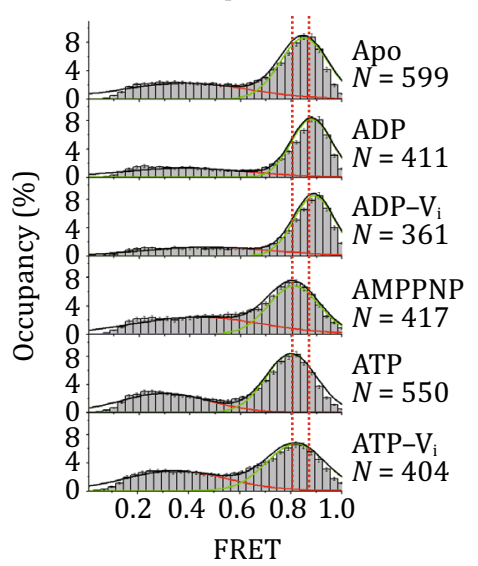

C

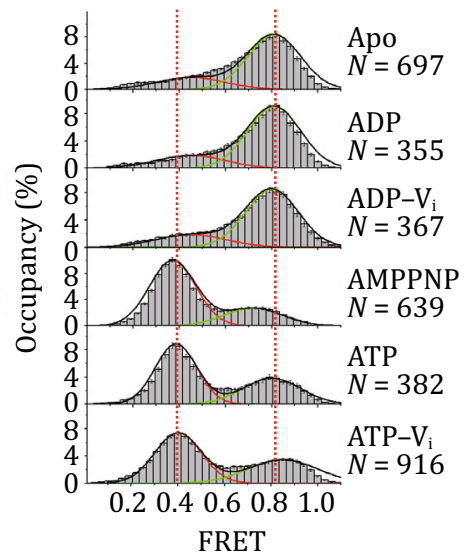

D

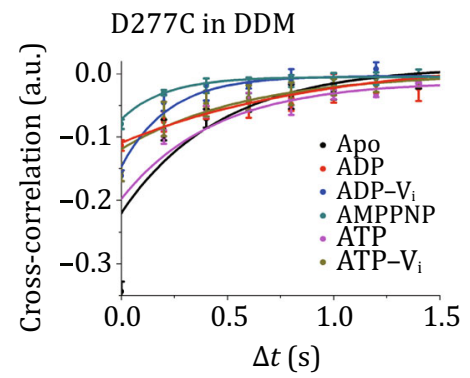

$\mathbf{E}$

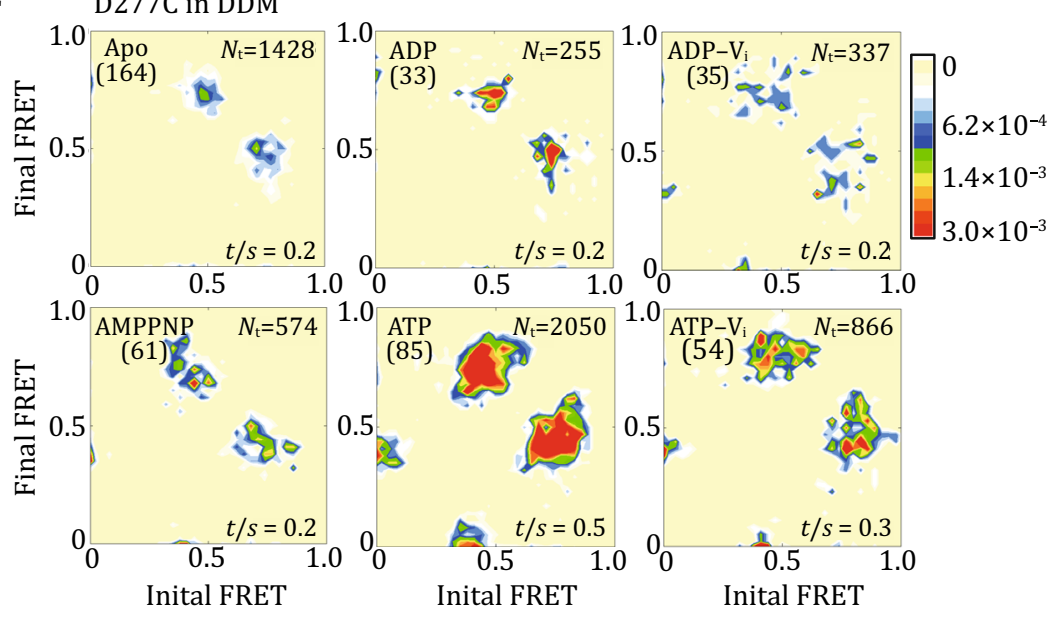

Fig. 4 Conformational dynamics of transmembrane domains (TMDs) of MsbA. A-C FRET histograms for MsbA-D277C reconstituted into nanodiscs (A), liposomes (B), and DDM (C), in apo and different ligand-binding ensembles. Error bars indicate standard deviations of 1000 bootstrap samples of the FRET traces. D Cross-correlation plots show slightly enhanced dynamics for all ensembles of MsbA-D277C in DDM compared with nanodiscs. Error bars indicate standard deviations of results from five independent experiments. E Transitiondensity plots of MsbA-D277C of apo and different nucleotide ensembles in DDM. A two-state transition was assumed (See Fig. 2F for legend)

between the donor- and acceptor-fluorescence trajectories in all ensembles (Supplemental Fig. S4). The transition-density plot provided additional evidence of conformational rigidity of the TMDs.

In liposomes, the effects of different ligands on the distribution of FRET states resembled that in nanodiscs (Fig. 4B). Specifically, the $E_{\mathrm{FRET}}$-distribution peak centered at $\sim 0.9$ in the apo, ADP, and ADP- $V_{i}$ ensembles indicated a periplasmic-closed conformation. Similar to the case in nanodiscs, addition of AMPPNP, ATP, and ATP- $V_{i}$ to MsbA-D277C proteoliposomes induced a population shift toward lower FRET values, peaking at $\sim 0.8$. Notably, cross-correlation analysis showed that the amplitudes of negative correlations in the liposomes were slightly higher in all apo- and ligand-binding ensembles than those in nanodiscs (Supplemental Fig. S5A), yet they were lower than those obtained for the T561C variant in liposomes. This suggested that conformational transitions on the periplasmic side are less frequent than those on the cytoplasmic side. This notion is visualized in the transition-density plots (Supplemental Fig. S5B). Collectively, these results suggest that the TMDs of MsbA in liposomes are more mobile than those in nanodiscs.

In detergent micelles, unimodal FRET distributions peaking at $\sim 0.8$ were obtained in the apo, ADP, and ADP- $V_{i}$ ensembles of MsbA-D277C (Fig. 4C), indicating a periplasmic-closed conformation. Addition of AMPPNP, ATP, or ATP- $V_{i}$ to MsbA-D277C induced a shift to a low $E_{\text {FRET }}(\sim 0.4)$, and presumably resulted in a 
periplasmic-opened conformation(s). These data clearly demonstrated that the FRET-probes at D277C undergo a large environmental change that can be reported by smFRET. Together, these observations indicated that, in the presence of ATP or an ATP analog, an open conformation(s) on the periplasmic side of MsbA is populated. Cross-correlation analysis showed that, in DDM, the negative correlation amplitudes of the ATP and apo ensembles were higher than those of the other ensembles (Fig. 4D). Transition-density plots further indicated that frequent transitions occurred between states with $E_{\text {FRET }} \sim 0.8$ and $\sim 0.4$ (Fig. $4 \mathrm{E}$ ).

Together, comparison between data from the D277C and T561C variants showed that the dynamics on the periplasmic side are drastically different from those on the cytoplasmic side, and that in the experimental conditions used, movements on the two sides of MsbA are only qualitatively coupled.

\section{DISCUSSION}

Understanding the population distribution and transition dynamics between distinct states is critical to delineate the mechanisms of any $\mathrm{ABC}$ transporter. Usually, crystallographic studies provide snapshots of the structures of different states of the target protein one at a time, and cryo-EM studies may reveal static structural information of a few major states from one experiment. The smFRET technique complements such structural studies by adding information on both thermodynamic distributions and transition dynamics between states. According to the Boltzmann theorem, the population distribution of the target protein obtained from smFRET provides information about the relative free energy differences $(\Delta G)$ between states under either equilibrium (e.g. in the apo ensemble) or steady-state (e.g. in the ATP ensemble) conditions (see Supplemental Table S2); such states may or may not be accessible in structural studies. In addition, fluorescence trajectories from individual molecules reveal the transition dynamics (e.g., the transition rates) between states (Zhu et al. 2017), thus drawing connections between multiple states of a chemical-kinetics network of the target protein (e.g., that represented by the KingAltman plot).

In this study, we used smFRET to analyze the dynamics of MsbA during its ATP-hydrolysis cycle in three environmental conditions (i.e., in DDM micelles, liposomes, and nanodiscs). Two MsbA residues, T561 and D277, were individually mutated to cysteine and labeled with fluorescent dyes, and we successfully demonstrated that they could be used to probe the conformational changes of the NBDs and TMDs, respectively, of MsbA. In particular, the T561C variant revealed three major clusters of conformational states (with ${ }^{\mathrm{T} 561 \mathrm{C}} E_{\mathrm{FRET}} \sim 0.2, \sim 0.6$, and $\sim 0.9$ ) in the cytoplasmic region (Fig. 2), whereas the D277C variant revealed two such clusters $\left({ }^{\mathrm{D} 277 \mathrm{C}} E_{\mathrm{FRET}} \sim 0.4\right.$ and $\sim 0.8$ ) in the periplasmic region (Fig. 4). Based on available structural information, it is reasonable to assume that the states with ${ }^{\mathrm{T} 561 \mathrm{C}} E_{\mathrm{FRET}} \sim 0.2, \sim 0.6$, and $\sim 0.9$ correspond to inward-facing (cytoplasmic-open), intermediate (cytoplasmic semiclosed), and outward-facing (cytoplasmic-closed) states, respectively (Fig. 5). Importantly, transition-density plots (e.g., Fig 3c) reveal that the transition from the inward-facing state to the outward-facing state must go through the intermediate state. Whereas the periplasmic-closed state $\left({ }^{\mathrm{D} 277 \mathrm{C}} E_{\mathrm{FRET}}\right.$ $\sim 0.8$ ) seems to correspond to the cytoplasmic-open state $\left({ }^{\mathrm{T} 561 \mathrm{C}} E_{\mathrm{FRET}} \sim 0.2\right)$, the periplasmic-open state ( ${ }^{\mathrm{D} 277 \mathrm{C}} E_{\text {FRET }} \sim 0.4$ ) is likely to correspond to the cytoplasmic-closed state $\left({ }^{\mathrm{T} 561 \mathrm{C}} E_{\mathrm{FRET}} \sim 0.9\right)$ and probably the cytoplasmic semiclosed state $\left({ }^{\mathrm{T} 561 \mathrm{C}} E_{\mathrm{FRET}} \sim 0.6\right)$ as well. Thus, FRET-probes at the periplasmic D277C site seem unable to decisively distinguish the intermediate state from the outward-facing state.

By analyzing smFRET data for the T561C variant (mutated in the NBD), we conclude that, in nanodiscs, MsbA is restricted to conformations in which the two NBDs remain close to each other (with ${ }^{\mathrm{T} 561 \mathrm{C}} E_{\mathrm{FRET}}$ $\sim 0.6$ ), in agreement with the cryo-EM structures of nanodisc-embedded MsbA (Mi et al. 2017), as well as the results of our ATPase-activity assays (Fig. 1C). Upon addition of AMPPNP, ATP, or ATP- $V_{i}$, the population peak shifted slightly toward higher FRET values (from 0.6 to 0.65 in nanodiscs) (Fig. 2A). Moreover, in the ATP ensemble, conformational transitions mainly occurred between states with ${ }^{\mathrm{T} 561 \mathrm{C}} E_{\mathrm{FRET}} \sim 0.3$ and $\sim 0.65$ (Fig. 2F). The average transition frequency of the conformational cycle $\left(\sim 0.1 \mathrm{~s}^{-1}\right)$ further suggests that the transporter is only "occasionally" pushed from the intermediate state $\left({ }^{\mathrm{T} 561 \mathrm{C}} E_{\mathrm{FRET}} \sim 0.65\right)$ into the higherenergy inward-facing state $\left({ }^{\mathrm{T} 561 \mathrm{C}} E_{\mathrm{FRET}} \sim 0.3\right)$ by a power stroke of ATP hydrolysis. However, comparing with the $25 \mathrm{~s}^{-1}$ rate of ATP hydrolysis in nanodiscs, the transition rate of MsbA in the presence of ATP is $\sim 200$ fold slower. This rate difference suggests the possibility that the in vitro ATP hydrolysis cycle does not require a large conformational change (e.g., between ${ }^{\mathrm{T} 561 \mathrm{C}} E_{\text {FRET }} \sim 0.3$ and $\sim 0.65$ ); nevertheless, the energy release from ATP hydrolysis may stimulate conformational changes from time to time.

The restriction from the nanodisc construction appears to be alleviated in both proteoliposomes and detergent micelles. In contrast to nanodiscs, in both the 


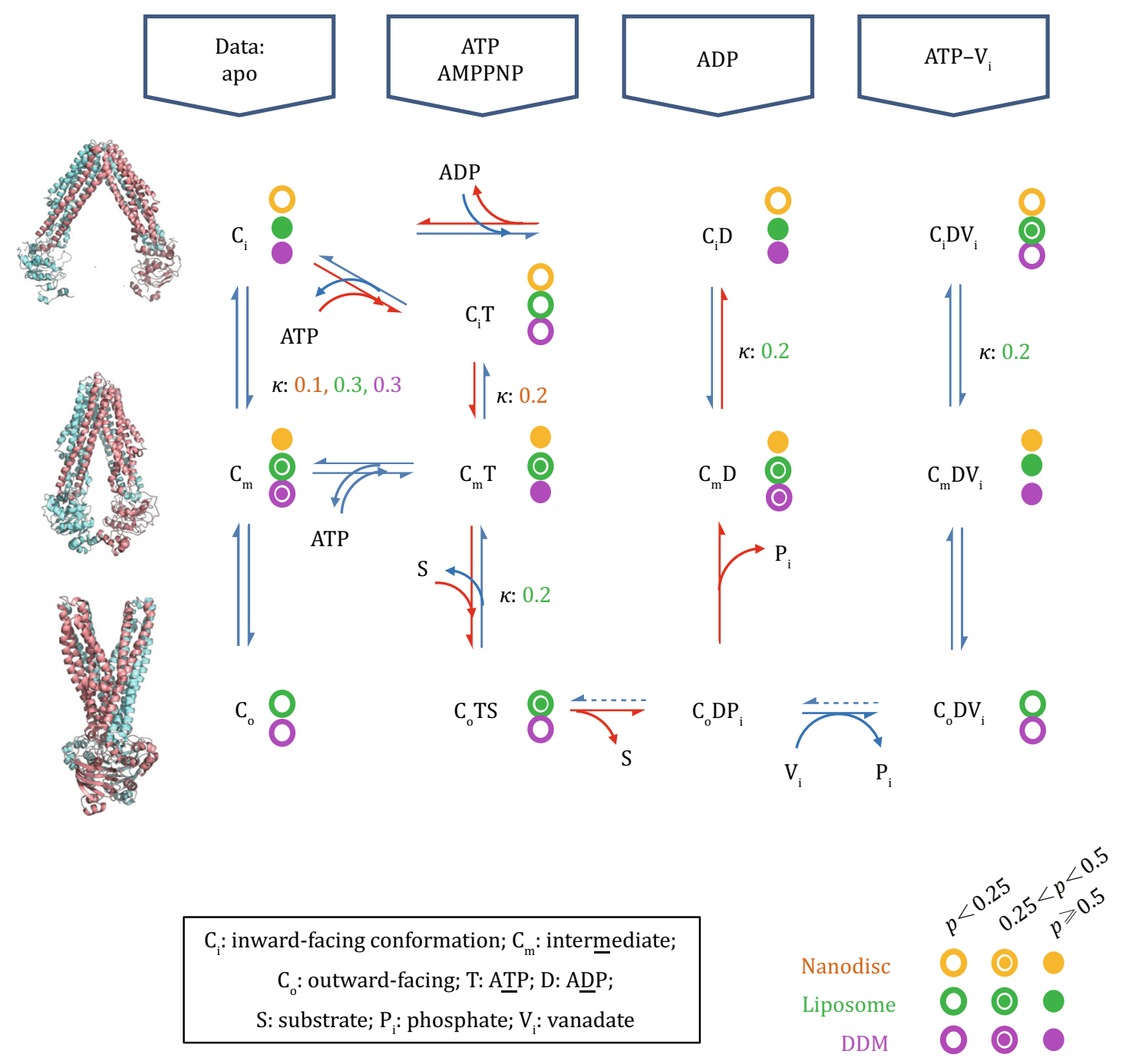

Fig. 5 King-Altman plot of the conformational transitions of MsbA. Transitions are labeled with arrows. The "canonical" transport cycle is shown with red arrows. Structures of the inward-facing (PDB ID: 3B5 W), intermediate (5TV4), and outward-facing (3B60) states are shown in ribbon presentation. Data on population probabilities $(p)$ is taken from Supplemental Table S2, and represented by circles: open, $p<0.25$; open with a dot, $0.25<p<0.5$; filled, $p \geq 0.5$. Data obtained in nanodiscs, liposomes, and DDM are colored orange, green, and magenta, respectively. Average rate $(\kappa)$ of one-way transitions is copied from the transition-density plots

liposomes and DDM, a wide-opening conformation of the NBD dimer with a unimodal distribution peaking at ${ }^{\mathrm{T} 561 \mathrm{C}} E_{\mathrm{FRET}} \sim 0.2$ was observed in the apo ensemble. Thus, in vivo, MsbA is likely to assume an inward-facing conformation before binding ATP. In addition to the intermediate state $\left({ }^{\mathrm{T} 561 \mathrm{C}} E_{\mathrm{FRET}} \sim 0.6\right)$, a more compact conformation $\left({ }^{\mathrm{T} 661 \mathrm{C}} E_{\mathrm{FRET}} \sim 0.9\right)$ was also captured in the AMPPNP and ATP ensembles. This compact state, which can be formed by the NBD dimers of MsbA in both the liposome and DDM conditions, seems to become inaccessible in the nanodiscs. In agreement with previous reports (Borbat et al. 2007; Cooper and Altenberg 2013; Zou et al. 2009; Zou and McHaourab 2010), these observations indicate that large-amplitude conformational dynamics can be adopted by MsbA in liposomes and DDM. Despite the apparently similar population-distributions of MsbA-T561C in the liposomes and DDM (Fig. 2), the NBD dimer of MsbA seems more dynamic in the liposomes (Fig. 3C), which is presumably closer to the native membrane environment, than in DDM or nanodiscs. In addition, under all the three conditions (i.e., nanodiscs, liposomes, and DDM), upon adding ADP, no change of conformation distribution was observed relative to the apo ensemble, in agreement with previous reports (Doshi et al. 2010; Mi et al. 2017; Weng et al. 2010). Curiously, the outwardfacing $\left({ }^{\mathrm{T} 561 \mathrm{C}} E_{\mathrm{FRET}} \sim 0.9\right)$ as well as the intermediate $\left.{ }^{\mathrm{T} 561 \mathrm{C} C} E_{\mathrm{FRET}} \sim 0.6\right)$ states observed in the ATP-V 
ensemble in both the liposomes and DDM become less accessible in the ADP- $V_{i}$ ensemble, indicating that in the presence of $\mathrm{V}_{\mathrm{i}}$, ATP hydrolysis traps MsbA in an "end"state that is not in equilibrium with the ADP-bound state(s) (which may even not bind $\mathrm{V}_{\mathrm{i}}$ ).

By means of the D277C variant, we also found that the transmembrane region of MsbA is less dynamic than the NBDs, especially in the liposomes and nanodiscs. Under all the three conditions (i.e., nanodiscs, liposomes, and DDM), MsbA adopts a periplasmic-closed conformation ( ${ }^{\mathrm{D} 277 \mathrm{C}} E_{\mathrm{FRET}} \sim 0.9$ ) in the apo, ADP, and $A D P-V_{i}$ ensembles (Fig. 4). However, in the AMPPNP, ATP, and ATP- $V_{i}$ ensembles, MsbA assumed a periplasmic-opened conformation $\left({ }^{\mathrm{D} 277 \mathrm{C}} E_{\mathrm{FRET}} \sim 0.4\right)$ in DDM. This trend was also observed in the liposomes, with the nanodiscs being the most restrictive condition. In addition, in DDM, the apo ensemble showed frequent transitions both between ${ }^{\mathrm{D} 277 \mathrm{C}} E_{\mathrm{FRET}} \sim 0.8$ and $\sim 0.4$ and between ${ }^{\mathrm{T} 561 \mathrm{C}} E_{\mathrm{FRET}} \sim 0.2$ and $\sim 0.6$ (Fig. $4 \mathrm{E}$ and Supplemental Fig. S3C), supporting the notion that whereas the periplasmic-closed conformation corresponds to the cytoplasmic-opened (inward-facing) state, the periplasmic-opened conformation corresponds to the intermediate state. Moreover, in DDM, the average rate of the conformational cycles of the periplasmic region of the TMD dimer in the presence of ATP was $<0.25 \mathrm{~s}^{-1}$, comparable with the ATP-hydrolysis rate of $3 \mathrm{~s}^{-1}$ per MsbA molecule. Again, the rate difference indicates that ATP hydrolysis and the cycle of conformational changes of the TMD dimer are (partially) decoupled in the experimental conditions used here. More generally, our current study strongly suggests that the choice of solubilization method markedly affects the thermodynamic distribution of states and kinetic behaviors of membrane proteins. The nanodisc construction strongly restricts the dynamics of MsbA on both sides of the lipid bilayer, whereas DDM micelles allow for wider opening of the periplasmic side of MsbA than liposomes and nanodiscs. Therefore, potential effects of the solubilization method(s) should be considered when interpreting results from in vitro studies of membrane proteins, especially concerning their dynamic behaviors.

The functional cycle of MsbA follows the general alternating-access model of transporters (Jardetzky 1966). A putative chemical-kinetics network (i.e., a King-Altman plot) of MsbA is shown in Fig. 5, in which the "canonical" functional cycle is embedded (as red arrows). On the one hand, our current study sheds new light on the function of MsbA by providing thermodynamic and kinetic data to describe this network in detail. On the other hand, an ideal ABC transporter sitting in the cellular membrane would not continuously consume ATP in the absence of substrates. Therefore, a missing piece of the picture of the MsbA functional cycle from the current study seems to be how the binding of substrate triggers ATP hydrolysis. Such a mechanism may be related to factors that have not been included in the currently used in vitro model systems. For example, the electrochemical potential of protons has been proposed to play certain roles in MsbA (Singh et al. 2016). Lacking a proper membrane potential-which may result in a shift of the equilibrium position and orientation of electrically charged membrane proteins relative to the membrane-may explain the apparent decoupling of ATP hydrolysis from substrate transport in MsbA in the current study. Future investigations, for example, of smFRET in the presence of a membrane potential, will verify such mechanistic hypotheses.

\section{MATERIALS AND METHODS}

\section{Protein expression and purification}

The gene of Cys-less MsbA (C88A/C315A, WT*) (Cooper and Altenberg 2013; Moeller et al. 2015) was synthesized commercially with $\mathrm{NdeI} / \mathrm{BamHI}$ restriction sites. It was cloned into vector pET-19b containing an Nterminal $\mathrm{His}_{10}$-tag. The WT MsbA and single point Cys mutants D277C and T561C were (back-) generated with WT* MsbA as the template using the QuikChange Mutagenesis Kit (Stratagene, CA).

The pET-19b plasmids with different MsbA-coding DNAs were transformed into $E$. coli strain C43. The cells were cultured in Terrific Broth to $\mathrm{OD}_{600} 0.8$, and induced using $0.5 \mathrm{mmol} / \mathrm{L}$ isopropyl $\beta$-D-thiogalactoside at $16{ }^{\circ} \mathrm{C}$ for $16 \mathrm{~h}$. Cells were collected and resuspended in buffer A (20 mmol/L HEPES (pH 7.5), $300 \mathrm{mmol} / \mathrm{L}$ $\mathrm{NaCl}, 5 \mathrm{mmol} / \mathrm{L} \beta$-mercaptoethanol, and $10 \% \mathrm{(v/}$ $v$ ) glycerol) and disrupted at 10,000-15,000 p.s.i. using a JN-R2C homogenizer (JNBio, China). Whole cells and cell debris were removed by centrifugation at $17,000 \mathrm{~g}$ for $10 \mathrm{~min}$, and the supernatant was ultracentrifuged at $100,000 \mathrm{~g}$ for $1 \mathrm{~h}$. The membrane fraction collected from the pellet was solubilized in buffer A supplemented with $1 \%(w / v)$ DDM (Anatrace, $\mathrm{OH}$ ) for $1 \mathrm{~h}$ at $4{ }^{\circ} \mathrm{C}$. After a second ultracentrifugation at $100,000 \mathrm{~g}$ for $30 \mathrm{~min}$, the supernatant was loaded onto the $\mathrm{Ni}^{2+}$-nitrilotriacetate affinity column (Ni-NTA; Qiagen, IL) and washed with buffer A containing $60 \mathrm{mmol} / \mathrm{L}$ imidazole and $0.05 \%$ $(w / v)$ DDM. The protein sample was eluted with buffer B $(20 \mathrm{mmol} / \mathrm{L}$ HEPES (pH 7.5), $100 \mathrm{mmol} / \mathrm{L} \mathrm{NaCl}$, and $10 \%(\mathrm{v} / \mathrm{v})$ glycerol) containing $300 \mathrm{mmol} / \mathrm{L}$ imidazole and $0.05 \%(w / v)$ DDM. The concentrated sample was then loaded onto a Superdex-200 10/30 column (GE 
Healthcare) pre-equilibrated with buffer B containing $0.05 \%(w / v)$ DDM.

\section{MsbA labeling}

Protein samples $(20 \mu \mathrm{mol} / \mathrm{L})$ were labeled in buffer B containing $0.05 \%(w / v)$ DDM, $200 \mu \mathrm{mol} / \mathrm{L}$ Cy3 dye, and $200 \mu \mathrm{mol} / \mathrm{L}$ Cy5 maleimide (GE Healthcare) for $30 \mathrm{~min}$ at $4{ }^{\circ} \mathrm{C}$. Free dyes were removed by loading the samples onto a Zeba ${ }^{\mathrm{TM}}$ Spin desalting column (Thermo Scientific). Labeling efficiency measurement was carried out with protein labeled by Cy5 maleimide. The extent of the labeling was estimated from absorption spectra of labeled protein by measuring peak maxima at $649 \mathrm{~nm}$ using a NanoPhotometer P330 (IMPLEN, Germany). The labeled-protein concentration was measured using a Bicinchoninic Acid Protein Assay Kit (CWBIO, China). The labeling efficiency at all selected mutation sites was $>90 \%$.

\section{Reconstitution of MsbA into liposomes}

E. coli total lipid extract (Avanti, AL) was dissolved at $20 \mathrm{mg} / \mathrm{mL}$ in buffer C (20 mmol/L HEPES (pH 7.5), $100 \mathrm{mmol} / \mathrm{L} \mathrm{NaCl}$ ). Before reconstitution, the lipid was extruded through a $0.4 \mu \mathrm{m}$ filter $>11$ times. The lipid bilayers were destabilized by the addition of $1 \%(w /$ $v$ ) DDM for $1 \mathrm{~h}$ at room temperature. For reconstitution for smFRET experiments, a labeled protein sample was added to lipids at a final protein:lipid ratio of 1:1000 $(w / w)$ and incubated for $30 \mathrm{~min}$ at room temperature. Prewashed and equilibrated Bio-Beads (Bio-Rad, Inc.) were added to the proteoliposome mixtures at $100 \mathrm{mg} / \mathrm{mL}$ and incubated at $4{ }^{\circ} \mathrm{C}$ for $1 \mathrm{~h}$. Then, the beads were replaced twice and incubated with the proteoliposome suspensions overnight at $4{ }^{\circ} \mathrm{C}$. The multilayer vesicles were harvested by centrifugation at $320,000 \mathrm{~g}$ for $20 \mathrm{~min}$ (Georgieva et al. 2013). Before imaging, liposomes were extruded through a $0.1 \mu \mathrm{m}$ filter $>21$ times to obtain homogenously sized proteoliposomes. Then, the liposomes were loaded onto Ni-NTA, and the proteoliposomes oriented with NBDs facing outward were eluted and obtained.

For activity assays, liposomes were prepared by the same method, except the ratio of protein to lipid was 1:5 $(w / w)$. Liposomes were extruded through a $0.4 \mu \mathrm{m}$ filter $>11$ times to obtain homogenously sized liposomes.

\section{Reconstitution of MsbA into nanodiscs}

Membrane scaffold protein MSP1E3D1 was purified as previously described (Ritchie et al. 2009). Purified and labeled MsbA was prepared as described above. For reconstitution, $68 \mu \mathrm{l} E$. coli total lipid (20 $\mathrm{mg} / \mathrm{mL}$ ) and $34 \mu$ biotin-phosphatidylethanolamines (PE) $(1 \mathrm{mg} / \mathrm{mL})$ were added to $500 \mu \mathrm{l}$ buffer $\mathrm{C}$, and then MSP1E3D1 and MsbA were added with molar ratio MSP1E3D1:lipid:MsbA of 2:120:1. The reconstitution mixtures were rotated at $4{ }^{\circ} \mathrm{C}$ for $1 \mathrm{~h}$, then prewashed and equilibrated Bio-Beads were added to the mixtures at $300 \mathrm{mg} / \mathrm{mL}$. The beads were incubated overnight with the mixture suspensions at $4{ }^{\circ} \mathrm{C}$. The aggregation of MsbA and empty nanodiscs was removed by loading the sample onto a Superdex-200 10/30 column.

\section{ATPase-activity measurements}

The ATPase activity of MsbA was measured by the release of $P_{i}$ from ATP using the malachite green colorimetric assay (Venter et al. 2008). Standards containing 0-7 nmol $\mathrm{P}_{\mathrm{i}}$ were prepared by dilution of $1 \mathrm{~mol} / \mathrm{L}$ potassium phosphate buffer ( $\mathrm{pH} 7.5$ ) with concentrations from 0 to $140 \mu \mathrm{mol} / \mathrm{L}$ in $20 \mu \mathrm{mol} / \mathrm{L}$ increments in a final volume of $50 \mu \mathrm{l}$. Purified protein $(1 \mu \mathrm{g})$ and reconstituted liposomes and nanodiscs $(0.1 \mu \mathrm{g})$ were added to $50 \mu \mathrm{l}$ of buffer $\mathrm{B}$ containing $5 \mathrm{mmol} / \mathrm{L} \mathrm{MgSO}_{4}$ and $10 \mathrm{mmol} / \mathrm{L}$ ATP with or without $0.05 \%(w / v)$ DDM respectively, followed by incubation for $3 \mathrm{~min}$ at $37^{\circ} \mathrm{C}$. The ATPase reaction was terminated by adding $300 \mu \mathrm{l}$ fresh malachite green solution, then samples were incubated for $5 \mathrm{~min}$ at $20^{\circ} \mathrm{C}$. Finally, $150 \mu \mathrm{l}$ of $34 \%$ $(w / v)$ citric acid was added to each sample, followed by incubation for $30 \mathrm{~min}$ at $30{ }^{\circ} \mathrm{C}$ in the dark to allow color development. The samples were measured at $600 \mathrm{~nm}$ using a NanoPhotometer P330.

\section{Determination of MsbA orientation in liposomes in activity assay}

The functional mutant MsbA-T561C was reconstituted into proteoliposomes and labeled with $\mathrm{Cy} 5$ maleimide. Non-disrupted and disrupted proteoliposome (1\% DDM) samples were analyzed by SDS-PAGE and using a fluorescence gel scanner (Tanon, China). The ratio of MsbA with NBDs facing outside was calculated based on comparison of the band fluorescence intensities using ImageJ software. The analysis showed that about $53 \% \pm 1 \%$ of the MsbA molecules were oriented with the NBDs facing outside.

\section{Single-molecule FRET imaging experiments}

SmFRET imaging experiments were carried out as previously described (Zhao et al. 2010b). Imaging chambers passivated with a mixture of polyethylene glycol (PEG) and biotin-PEG were incubated with $100 \mu \mathrm{g} / \mathrm{mL}$ 
streptavidin. Two methods of imaging were used. (1) For MsbA in detergent, His $_{10}$-tagged dye-labeled samples were immobilized onto the streptavidin-treated chamber surface via biotin-NTA- $\mathrm{Ni}^{2+}$. They were imaged in buffer B containing $0.05 \%(w / v)$ DDM. (2) For MsbA reconstituted into nanodiscs and liposomes, samples were immobilized onto the streptavidin-treated chamber surface using biotin-PE, and the imaging experiments were carried out in buffer B. All ensembles with nucleotides were assayed in the presence of additional $5 \mathrm{mmol} / \mathrm{L} \mathrm{MgCl}_{2}$. To prolong the lifetime of the fluorescence, an oxygen-scavenging system $\quad 0.1 \%$ glucose, $5 \mathrm{mmol} / \mathrm{L} \quad \beta$-mercaptoethanol, $1 \mathrm{unit} / \mathrm{mL}$ glucose oxidase, $1 \mathrm{unit} / \mathrm{mL}$ catalase, and $1 \mathrm{mmol} / \mathrm{L}$ cyclo-octatetraene) was added into the imaging buffer. Images were taken at $50 \mathrm{~ms} /$ frame.

Fluorescence signals were collected by means of an objective-based total internal reflection fluorescent microscope, and data were acquired using the software Metamorph (Universal Imaging Corporation) (Zhao et al. 2010b). Data analysis was performed as described in previous studies (Juette et al. 2016). All assays were repeated at least three times, and the results were reproducible.

\section{Steady-state fluorescence anisotropy measurements}

To verify low anisotropy of the labeled protein samples, steady-state anisotropy measurements of Cy5-labeled MsbA (10 nmol/L) were carried out using a Hitachi F-7000 spectrofluorometer in buffer B containing $0.05 \%(w / v)$ DDM. The excitation and emission wavelengths were 646 and $662 \mathrm{~nm}$, respectively. The results confirmed that dyes attached to MsbA variants with different nucleotides bound had low anisotropy (Supplemental Table S1).

$\begin{array}{ll}\text { Abbreviations } \\ \text { ABC } & \text { ATP-binding cassette (transporters) } \\ \text { ADP } & \text { Adenosine diphosphate } \\ \text { AMPPNP } & \beta, \gamma \text {-imido-adenosine } 5^{\prime} \text {-triphosphate } \\ \text { ATP } & \text { Adenosine triphosphate } \\ \text { DDM } & \text { n-dodecyl- } \beta \text {-D-maltopyranoside } \\ \text { NBD } & \text { Nucleotide-binding domain } \\ \text { TMD } & \text { Transmembrane domain }\end{array}$

Acknowledgements This study was supported by the National Basic Research Program of China (973 Program) (2014CB910400) and the National Natural Science Foundation of China (31522016 and 31470745). We thank James Allen, DPhil, from Liwen Bianji,
Edanz Group China, for editing the English text of a draft of this manuscript.

\section{Compliance with Ethical Standards}

Conflict of interest Yanqing Liu, Yue Liu, Lingli He, Yongfang Zhao, and Xuejun C. Zhang declare that they have no conflict of interest.

Human and animal rights and informed consent This article does not contain any studies with human or animal subjects performed by any of the authors.

Open Access This article is distributed under the terms of the Creative Commons Attribution 4.0 International License (http:// creativecommons.org/licenses/by/4.0/), which permits unrestricted use, distribution, and reproduction in any medium, provided you give appropriate credit to the original author(s) and the source, provide a link to the Creative Commons license, and indicate if changes were made.

\section{References}

Borbat PP, Surendhran K, Bortolus M, Zou P, Freed JH, Mchaourab HS (2007) Conformational motion of the ABC transporter MsbA induced by ATP hydrolysis. PLoS Biol 5:e271

Cooper RS, Altenberg GA (2013) Association/dissociation of the nucleotide-binding domains of the ATP-binding cassette protein MsbA measured during continuous hydrolysis. J Biol Chem 288:20785-20796

Dassa E (2011) Natural history of ABC systems: not only transporters. Essays Biochem 50:19-42

Davidson AL (2002) Mechanism of coupling of transport to hydrolysis in bacterial ATP-binding cassette transporters. J Bacteriol 184:1225-1233

Davidson AL, Chen J (2004) ATP-binding cassette transporters in bacteria. Annu Rev Biochem 73:241-268

Doerrler WT, Raetz CR (2002) ATPase activity of the MsbA lipid flippase of Escherichia coli. J Biol Chem 277:36697-36705

Doerrler WT, Reedy MC, Raetz CR (2001) An Escherichia coli mutant defective in lipid export. J Biol Chem 276:11461-11464

Doerrler WT, Gibbons HS, Raetz CR (2004) MsbA-dependent translocation of lipids across the inner membrane of Escherichia coli. J Biol Chem 279:45102-45109

Dong J, Yang G, McHaourab HS (2005) Structural basis of energy transduction in the transport cycle of MsbA. Science 308:1023-1028

Doshi R, Woebking B, van Veen HW (2010) Dissection of the conformational cycle of the multidrug/lipidA $\mathrm{ABC}$ exporter MsbA. Proteins 78:2867-2872

Eckford PD, Sharom FJ (2008) Functional characterization of Escherichia coli MsbA: interaction with nucleotides and substrates. J Biol Chem 283:12840-12850

Georgieva ER, Borbat PP, Ginter C, Freed JH, Boudker O (2013) Conformational ensemble of the sodium-coupled aspartate transporter. Nat Struct Mol Biol 20:215-221

Higgins CF (2007) Multiple molecular mechanisms for multidrug resistance transporters. Nature 446:749-757

Higgins CF, Linton KJ (2004) The ATP switch model for ABC transporters. Nat Struct Mol Biol 11:918-926

Jardetzky O (1966) Simple allosteric model for membrane pumps. Nature 211:969-970 
Juette MF, Terry DS, Wasserman MR, Altman RB, Zhou Z, Zhao H, Blanchard SC (2016) Single-molecule imaging of nonequilibrium molecular ensembles on the millisecond timescale. Nat Methods 13:341-344

Lerner E, Cordes T, Ingargiola A, Alhadid Y, Chung SY, Michalet X, Weiss S (2018) Toward dynamic structural biology: two decades of single-molecule Forster resonance energy transfer. Science. https://doi.org/10.1126/science.aan1133

Locher KP (2016) Mechanistic diversity in ATP-binding cassette $(A B C)$ transporters. Nat Struct Mol Biol 23:487-493

McKinney SA, Joo C, Ha T (2006) Analysis of single-molecule FRET trajectories using hidden markov modeling. Biophys J 91:1941-1951

Mi W, Li Y, Yoon SH, Ernst RK, Walz T, Liao M (2017) Structural basis of MsbA-mediated lipopolysaccharide transport. Nature 549:233-237

Moeller A, Lee SC, Tao H, Speir JA, Chang G, Urbatsch IL, Potter CS, Carragher B, Zhang Q (2015) Distinct conformational spectrum of homologous multidrug $\mathrm{ABC}$ transporters. Structure 23:450-460

Rigaud J-L, Pitard B, Levy D (1995) Reconstitution of membrane proteins into liposomes application to energy-transducing membrane proteins. Biochim Biophys Acta 1231:223-246

Ritchie TK, Grinkova YV, Bayburt TH, Denisov IG, Zolnerciks JK, Atkins WM, Sligar SG (2009) Reconstitution of membrane proteins in phospholipid bilayer nanodiscs. Methods Enzymol 464:211-231

Ruiz N, Kahne D, Silhavy TJ (2009) Transport of lipopolysaccharide across the cell envelope: the long road of discovery. Nat Rev Microbiol 7:677-683

Singh H, Velamakanni S, Deery MJ, Howard J, Wei SL, van Veen HW (2016) ATP-dependent substrate transport by the ABC transporter MsbA is proton-coupled. Nat Commun 7:12387

Smith CA, Rayment I (1996) X-ray structure of the magnesium(II)ADP-vanadate complex of the dictyostelium discoideum Myosin Motor Domain to $1.9 \AA$ resolution. Biochemistry 35:5404-5417

Urbatsch IL, Tyndall GA, Tombline G, Senior AE (2003) P-glycoprotein catalytic mechanism: studies of the ADP-vanadate inhibited state. J Biol Chem 278:23171-23179
Vafabakhsh R, Levitz J, Isacoff EY (2015) Conformational dynamics of a class C G-protein-coupled receptor. Nature 524:497-501

Venter H, Velamakanni S, Balakrishnan L, van Veen HW (2008) On the energy-dependence of Hoechst 33342 transport by the ABC transporter LmrA. Biochem Pharmacol 75:866-874

Ward A, Reyes CL, Yu J, Roth CB, Chang G (2007) Flexibility in the $\mathrm{ABC}$ transporter MsbA: alternating access with a twist. Proc Natl Acad Sci USA 104:19005-19010

Weng JW, Fan KN, Wang WN (2010) The conformational transition pathway of ATP binding cassette transporter MsbA revealed by atomistic simulations. J Biol Chem 285:3053-3063

Zhang XC, Han L, Zhao Y (2016) Thermodynamics of ABC transporters. Protein cell 7:17-27

Zhao Y, Quick M, Shi L, Mehler EL, Weinstein H, Javitch JA (2010a) Substrate-dependent proton antiport in neurotransmitter: sodium symporters. Nat Chem Biol 6:109-116

Zhao Y, Terry D, Shi L, Weinstein H, Blanchard SC, Javitch JA (2010b) Single-molecule dynamics of gating in a neurotransmitter transporter homologue. Nature 465:188-193

Zhao Y, Terry DS, Shi L, Quick M, Weinstein H, Blanchard SC, Javitch JA (2011) Substrate-modulated gating dynamics in a $\mathrm{Na}^{+}$-coupled neurotransmitter transporter homologue. Nature 474:109-113

Zhou Z, White KA, Polissi A, Georgopoulos C, Raetz CR (1998) Function of Escherichia coli MsbA, an essential ABC family transporter, in lipid A and phospholipid biosynthesis. J Biol Chem 273:12466-12475

Zhu Y, Zhang L, Zhang XC, Zhao Y (2017) Structural dynamics of Gi $\alpha$ protein revealed by single molecule FRET. Biochem Biophys Res Commun 491:603-608

Zou P, Mchaourab HS (2010) Increased sensitivity and extended range of distance measurements in spin-labeled membrane proteins: Q-band double electron-electron resonance and nanoscale bilayers. Biophys J 98:L18-L20

Zou P, Bortolus M, McHaourab HS (2009) Conformational cycle of the $\mathrm{ABC}$ transporter MsbA in liposomes: detailed analysis using double electron-electron resonance spectroscopy. J Mol Biol 393:586-597 